\title{
User-Based Discrete-Time Queuing Analysis for Opportunistic Spectrum Access in Cognitive Radio Networks
}

\author{
Lei Zhang1,2, Shanzhi Mou', Yu Wang1 \\ ${ }^{1}$ School of Electrical and Information Engineering, Jiangsu University of Technology, Changzhou, China \\ ${ }^{2}$ National Mobile Communications Research Laboratory, Southeast University, Nanjing, China \\ ${ }^{3}$ School of Mathematics and Physics, Jiangsu University of Technology, Changzhou, China \\ Email: zhlei@jsut.edu.cn
}

How to cite this paper: Zhang, L., Mou, S.Z. and Wang, Y. (2019) User-Based Discrete-Time Queuing Analysis for Opportunistic Spectrum Access in Cognitive Radio Networks. Advances in Pure Mathematics, 9, 1-14.

https://doi.org/10.4236/apm.2019.91001

Received: December 10, 2018

Accepted: January 7, 2019

Published: January 10, 2019

Copyright $\odot 2019$ by author(s) and Scientific Research Publishing Inc. This work is licensed under the Creative Commons Attribution International License (CC BY 4.0).

http://creativecommons.org/licenses/by/4.0/

\begin{abstract}
In cognitive radio networks, the spectrum utilization can be improved by cognitive users opportunistically using the idle channels licensed to the primary users. However, the new arrived cognitive users may not be able to use the channel immediately since the channel usage state is random. This will impose additional time delay for the cognitive users. Excessive waiting delay can make cognitive users miss the spectrum access chances. In this paper, a discrete-time Markov queuing model from a macro point of view is provided. Through the matrix-geometric solution theory, the average sojourn time for cognitive users in the steady state before accessing the spectrum is obtained. Given the tolerant delay of cognitive users, the macro-based throughput is derived and an access control mechanism is proposed. The numerical results show the effects of service completion probability on average sojourn time and throughput. It is confirmed that the throughput can be obviously improved by using the proposed access control mechanism. Finally, the performance evaluations based on users are compared to that based on data packets.
\end{abstract}

\section{Keywords}

Cognitive Radio Networks, Macro-Level Spectrum Access, Discrete-Time Queuing Theory, Matrix-Geometric Solution, Access Control

\section{Introduction}

Cognitive radio (CR) is an important technique to improve spectrum utilization efficiency, which employs spectrum sensing, spectrum decision and spectrum 
management to ensure that the cognitive users (CUs) (or secondary users) can opportunistically use the idle channels which are licensed to primary users (PUs) to communicate [1]. In order to turn CR into a reality, the QoS of CUs should be also paid more attention, certainly the quality of service (QoS) of PUs should be firstly guaranteed. Before accessing the spectrum, CUs may have to wait in queuing. And perhaps after accessing the spectrum, CUs' communication connections can be interrupted by arrival PUs and perform spectrum handoff procedure to continue the connections. Regardless of either waiting in queuing or carrying out spectrum handoff, the delay will be inevitable. And the excess delay will result in bringing down the QoS or system capacity of CUs. Therefore, the studies have been emerged in large quantities on performance analysis for spectrum access and methods to alleviate the decreases of QoS and capacity.

The systematic exposition for opportunistic spectrum access in CR networks was firstly provided by [2]. The challenges of dynamic spectrum access in distributed CR networks were summarized in [3] [4]. The authors of [5] evaluated the performance of a dynamic multi-channel access protocol. In order to improve the capacity, the authors of [6] optimized spectrum sensing and decision uniformly by dynamic programming algorithm, while the authors in [7] proposed a joint resource allocation method for CR femtocell networks and in [8] presented three efficient algorithms to address the channel allocation problem for multi-channel cognitive vehicular networks. In [9], the tradeoff between spectrum efficiency and fairness was investigated for a dynamic resource allocation algorithm. There are also some other QoS-enhanced approaches which are generally performed when CUs access the spectrum. For example, in [10] and [11], the authors proposed reasonable spectrum handoff strategies to directly reduce the handoff delay caused by interruptions. For various bandwidth CR networks, the authors of [12] analyzed the handoff delay that usually occurs after accessing the spectrum.

One of the most effective and actual performance analysis method for opportunistic spectrum access and multiple spectrum handoff is based on dynamic Markov queuing model, which has been the popular issues in recent years [13] [14]. For example, the authors of [15] carried out a complex queuing analysis for spectrum utilization of PUs and CUs in CR cellular networks, and obtained the average packet queue length, the average packet waiting time and packet losing rate of CUs.

However, up to present, the performance analysis studies for opportunistic spectrum access of CUs are almost all based on data packet. It is no doubt that analyzing the spectrum mobility management based on packet level has contributed to design CR MAC protocol, however, the macro level-based capacity of a cognitive cell which can directly guide the deployment of CR access points (APs), especially in user experience-based wireless system. If the spectrum access is evaluated based on data packets (DP), the user may drop its connection and search another channel during its communications when the upper limit of packet delay is reached, which will reduce the user's experience. The spectrum 
access based on macroscopic users (MU) can reduce the packet delay so long as the users' upper limits of delay are not reached.

Accordingly, other than the DP-based queuing analysis, in this paper, we firstly model the opportunistic spectrum access process as a discrete-time Markov chain from the MU level, We evaluate the performance using discrete-time queuing theory. The average sojourn time of CUs in queue before accessing the spectrum is obtained through matrix-geometric solution theory. Considering each CU's tolerable delay, the expression of CU's throughput is given and an access control (AC) method is proposed to improve the throughput. In order to compare with the DP-based evaluation result, we define the transmission intact ratio, which is the ratio of the amount of users with no packets dropping and the amount of users which are allowed to access the spectrum. The numerical results show the effects of CUs' service completion probability on the average sojourn time and throughput in steady state. The throughput comparison between AC and without $\mathrm{AC}$ is provided, which indicates the throughput can be improved many fold using AC. Finally, the evaluation results based on MU are compared to that based on DP. The transmission intact ratio of MU-based spectrum access outperforms the DP-based. These analytical results can guide to design the spectrum access mechanism for CUs in CR networks.

The rest of the paper is organized as follows. Section 2 describes the system model. Section 3 gives the solution of the steady state probability. Section 4 analyzes the throughput and transmission intact ratio of the system. Section 5 shows the numerical results to evaluate the performance. Section 6 concludes this paper.

\section{System Model}

A CR network based on cell structure illustrated in Figure 1 is considered in this paper. A primary cell has a PU base station (PU BS) and a CR cell has a cognitive radio access point (CR AP). The primary system is a frequency division system, and one channel is licensed to one PU. The cognitive system adopts centralized collaboration spectrum sensing, each CU's sensing result should be sent to fusion center, the fusion center will use data fusion method to obtain the final decision result and then the information of decision result will be broadcasted to CUs in this cell, the CUs can decide to access the channel according to received decision result. Before allowed to access the channel, the CU with data transmitting requirement has to wait in the virtual queue of CR AP. It is assumed that the buffer capacity is infinite and the queue regulation is first-come first-serve (FCFS).

We analyze the traffic loads and spectrum access for PUs and CUs through random process. The spectrum is divided into different time slots (TS) in time indicated in Figure 2 for cognitive system. In each TS, there are one head-end denoted as $\left(n, n^{+}\right)$and one tail-end $\left(n^{-}, n\right) . n^{+}$and $n^{-}$are just two point marks in order to divide the TS more subtly. $\left(n, n^{+}\right)$and $\left(n^{-}, n\right)$ are two subminiature intervals. It is assumed that the arrivals for primary and cognitive users 


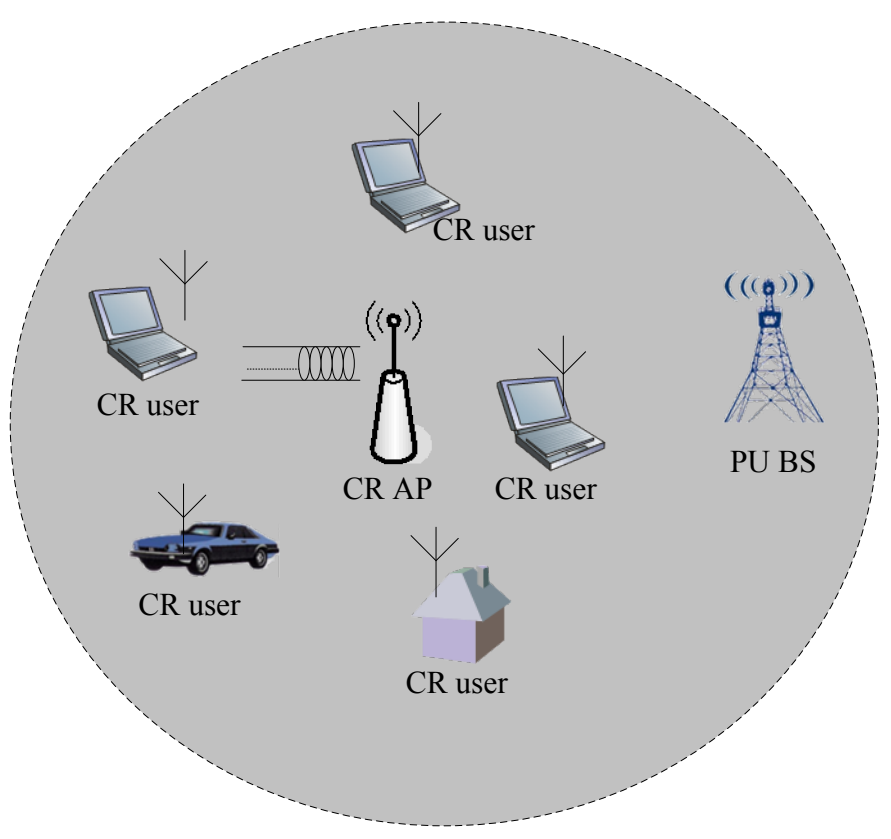

Figure 1. A cellular structure-based cognitive radio network.

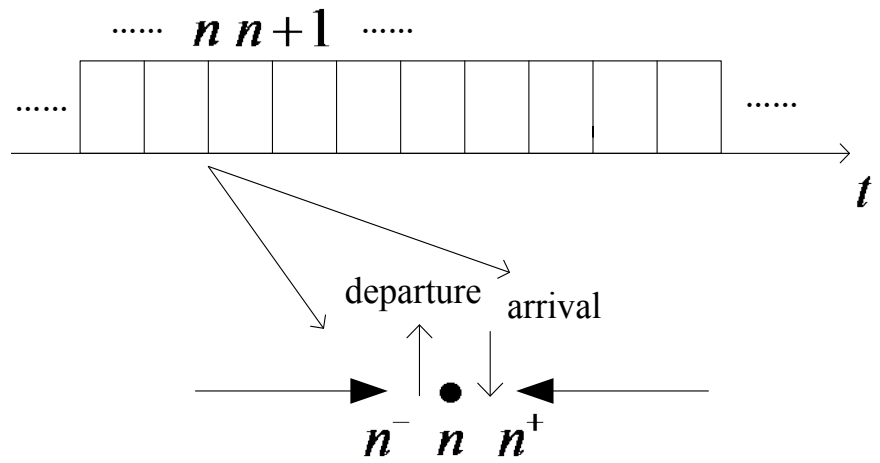

Figure 2. The description for arrival and departure of the user on single channel.

take place in head-end $\left(n, n^{+}\right)$and the departures take place in tail-end $\left(n^{-}, n\right)$. Here the users' arrival means the communication requirement is generated, and the departure means the communication requirement is dealt with (served). Since PUs have the preemptive priority to utilize the channel, the new arrived PU can use the channel immediately to communicate at the moment $n^{+}$(we ignore the handshaking time). The new arrived $\mathrm{CU}$ will have to wait in the queue until the on-going PU and all of the existing CUs finish their services. The beginning time of service is also moment $n^{+}$of a certain TS. Certainly, the on-going service of $\mathrm{CU}$ will be interrupted by the new arrived $\mathrm{PU}$, and the channel will be returned to the PU. We denote the channel state as " 1 " if the channel is occupied by PU, and " 0 " if the channel is not occupied by PU. If the PU arrives or departs, the channel state will change. The changing of channel state is assumed to occur at moment $n^{+}$. The following aim is to observe the spectrum state and the amount of CUs at moment $n^{+}$. 
Regard $\phi_{p} \quad\left(0<\phi_{p}<1\right)$ as the channel state transition probability from " 0 " to " 1 ", and $\bar{\phi}_{p}=1-\phi_{p}$ as the probability that the channel state stays at "0". In reverse, regard $\phi_{p s}\left(0<\phi_{p s}<1\right)$ as the channel state transition probability from " 1 " to " 0 ", and $\bar{\phi}_{p s}=1-\phi_{p s}$ as the probability that channel state stays at " 1 ". The channel state transition probability matrix can be given by

$$
\boldsymbol{D}=\left[\begin{array}{ll}
\bar{\phi}_{p} & \phi_{p} \\
\phi_{p s} & \bar{\phi}_{p s}
\end{array}\right]
$$

According to the state transition probability matrix, the channel state probabilities for " 0 " and “ 1 " are $\rho_{p}^{\text {off }}=\phi_{p s} /\left(\phi_{p s}+\phi_{p}\right)$ and $\rho_{p}^{o n}=\phi_{p} /\left(\phi_{p s}+\phi_{p}\right)$, respectively.

It is assumed that only one user can be served on one channel at one instant. The arrivals in each TS for CUs may be multiple or single, while departure is single, some other service-unfinished CUs have to wait in the queue. The amount of arrived CUs $\xi_{c}$ in each TS can be regarded as a non-negative integer random variable with probability distribution $\operatorname{Pr}\left\{\xi_{c}=k\right\}=\alpha_{k}, k \geq 0$ and $\sum_{k=0}^{\infty} \alpha_{k}=1$. The average arrival rate is $\lambda_{c}=\sum_{k=0}^{\infty} k \alpha_{k}$. The CU cannot be served

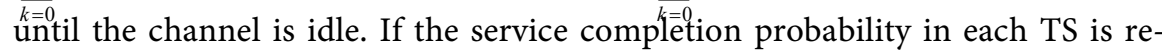
garded as $\mu_{c}$, the probability distribution of service TS can be given as $\operatorname{Pr}\left\{S_{c}=k\right\}=\bar{\mu}_{c}^{k-1} \mu_{c}, \bar{\mu}_{c}=1-\mu_{c}$, which is a geometric distribution with parameter $\mu_{c}, 0<\mu_{c}<1$.

Regard $L_{n}$ as the amount of CUs in system at moment $n^{+}$. Note that the arrived users in $\left(n, n^{+}\right)$should be counted in $L_{n}$. Define $J_{n} \in\{0,1\}$ as channel state. Hence, $\left\{\left(L_{n}, J_{n}\right), n \geq 1\right\}$ is regarded as embedded Markov chain for queuing process with state space $\Omega=\{(k, j) ; k \geq 0, j=0,1\}$. If the state transition probability for Markov chain is written by

$$
P_{(i, j)(k, l)}=\operatorname{Pr}\left\{L_{n+1}=k, J_{n+1}=l \mid L_{n}=i, J_{n}=j\right\},(i, j),(k, l) \in \Omega
$$

The system state transition probability matrix can be obtained by

$$
\boldsymbol{P}_{\mathrm{inf}}=\left[\begin{array}{ccccc}
\boldsymbol{A}_{0} & \boldsymbol{A}_{1} & \boldsymbol{A}_{2} & \boldsymbol{A}_{3} & \cdots \\
\boldsymbol{B}_{0} & \boldsymbol{B}_{1} & \boldsymbol{B}_{2} & \boldsymbol{B}_{3} & \cdots \\
\mathbf{0} & \boldsymbol{B}_{0} & \boldsymbol{B}_{1} & \boldsymbol{B}_{2} & \cdots \\
\mathbf{0} & \mathbf{0} & \boldsymbol{B}_{0} & \boldsymbol{B}_{1} & \cdots \\
\mathbf{0} & \mathbf{0} & \mathbf{0} & \ddots & \ddots
\end{array}\right]
$$

According to the above assumptions and definitions, each block sub-matrix in $\boldsymbol{P}_{\text {inf }}$ can be given as follows

$$
\begin{gathered}
\boldsymbol{A}_{i}=\boldsymbol{D} \alpha_{i} \\
\boldsymbol{B}_{i}=\left[\begin{array}{cc}
\left(\mu_{c} \alpha_{i}+\bar{\mu}_{c} \alpha_{i-1}\right) \bar{\phi}_{p} & \left(\mu_{c} \alpha_{i}+\bar{\mu}_{c} \alpha_{i-1}\right) \phi_{p} \\
\alpha_{i-1} \phi_{p s} & \alpha_{i-1} \bar{\phi}_{p s}
\end{array}\right]
\end{gathered}
$$

where $i=0,1,2,3, \cdots$. To form a common format, we set $\alpha_{-1}=0$ in $\boldsymbol{B}_{0}$.

To obtain the average sojourn time of CUs in steady state, the steady state probability for the amount of CUs should be given in advance. The next section 
provides the process to obtain the steady state probability through the theory of matrix-geometric solution.

\section{Solution for Steady State Probability}

Before you begin to format your paper, first write and save the content as a separate text file. Keep your text and graphic files separate until after the text has been formatted and styled. Do not use hard tabs, and limit use of hard returns to only one return at the end of a paragraph. Do not add any kind of pagination anywhere in the paper. Do not number text heads-the template will do that for you.

Finally, complete content and organizational editing before formatting. Please take note of the following items when proofreading spelling and grammar.

In order to obtain an accurate closed-form result, the following analysis is carried on a single channel and only one $\mathrm{CU}$ is allowed to enter into the queue at one TS. Multiple CUs' arrival can be extended in multiple-channel analysis. It would be well to assume that the $\mathrm{CU}$ will arrive at the queue or generate communication requirement in each TS as a probability $p_{c}$, and conversely not arrive as a probability $\bar{p}_{c}=1-p_{c}$. That is, the arrival interval TS for CU follows the geometric distribution with parameter $p_{c}$. Then we have $\operatorname{Pr}\left\{\xi_{c}=1\right\}=p_{c}$ and $\operatorname{Pr}\left\{\xi_{c}=0\right\}=\bar{p}_{c}$ with the arrival rate $\lambda_{c}=p_{c}$. As a consequence, the transition probability matrix, which is a special form of $\boldsymbol{P}_{\text {inf }}$ in (2), can be given by

$$
\tilde{\boldsymbol{P}}=\left[\begin{array}{ccccc}
\tilde{\boldsymbol{A}}_{0} & \tilde{\boldsymbol{A}}_{1} & \mathbf{0} & \mathbf{0} & \mathbf{0} \\
\tilde{\boldsymbol{B}}_{0} & \tilde{\boldsymbol{B}}_{1} & \tilde{\boldsymbol{B}}_{2} & \mathbf{0} & \mathbf{0} \\
\mathbf{0} & \tilde{\boldsymbol{B}}_{0} & \tilde{\boldsymbol{B}}_{1} & \tilde{\boldsymbol{B}}_{2} & \mathbf{0} \\
\mathbf{0} & \mathbf{0} & \ddots & \ddots & \ddots
\end{array}\right]
$$

where

$$
\begin{aligned}
& \tilde{\boldsymbol{A}}_{0}=\boldsymbol{D} \bar{p}_{c}, \tilde{\boldsymbol{A}}_{1}=\boldsymbol{D} p_{c}, \\
& \tilde{\boldsymbol{B}}_{0}=\left[\begin{array}{cc}
\mu_{c} \bar{p}_{c} \bar{\phi}_{p} & \mu_{c} \bar{p}_{c} \phi_{p} \\
0 & 0
\end{array}\right], \\
& \tilde{\boldsymbol{B}}_{1}=\left[\begin{array}{cc}
\left(\mu_{c} p_{c}+\bar{\mu}_{c} \bar{p}_{c}\right) \bar{\phi}_{p} & \left(\mu_{c} p_{c}+\bar{\mu}_{c} \bar{p}_{c}\right) \phi_{p} \\
\bar{p}_{c} \phi_{p s} & \bar{p}_{c} \bar{\phi}_{p s}
\end{array}\right], \\
& \tilde{\boldsymbol{B}}_{2}=\left[\begin{array}{cc}
\bar{\mu}_{c} p_{c} \bar{\phi}_{p} & \bar{\mu}_{c} p_{c} \phi_{p} \\
p_{c} \phi_{p s} & p_{c} \bar{\phi}_{p s}
\end{array}\right] .
\end{aligned}
$$

For acquiring the performance indexes in steady state of the queuing system, according to the discrete-time queuing theory [16], $p_{c}$ is assumed to be less than $\mu_{c}$ to insure the system can reach steady state. The matrix-geometric solution theory lets we know that the steady state probability $\pi$ of queue length for CUs is the unique solution of the following linear system of equations

$$
\left\{\begin{array}{l}
\boldsymbol{\pi} \cdot \tilde{\boldsymbol{P}}=\pi \\
\boldsymbol{\pi} \cdot \boldsymbol{e}=1
\end{array}\right.
$$

where $\boldsymbol{e}$ is a column vector with elements being ones, $\pi$ is a vector in the 
form of $\left[\pi_{0}, \pi_{1}, \pi_{2}, \cdots\right]$ where $\pi_{k}=\left[\pi_{k 0}, \pi_{k 1}\right]$ is a two-dimension row vector. $\pi_{k}(k \geq 0)$ represents the steady state probability when the queue length is $k$, and $\pi_{k j}(j=0,1)$ means the steady state probability when the queue length is $k$ and the channel state is $j$. As the discrete-Markov process is positive recurrence, the matrix-geometric solution and queuing theory can be used to derive the steady state probability $\pi$. The detail of deducing $\pi$ is provided in Appendix A where the key body is to solve the rate matrix $\boldsymbol{R}$.

\section{Throughput and Transmission Intact Ratio}

After obtaining the steady state probability, the average queue length $E[L]$ under steady state and the steady state probability $P_{p u}$ when channel is occupied by PU can be given as follows

$$
E[L]=\sum_{k=0}^{\infty} k\left(\pi_{k 0}+\pi_{k 1}\right), \quad P_{p u}=\sum_{k=0}^{\infty} \pi_{k 1}
$$

where $P_{p u}$ should be equal to the pre-given $\rho_{p}^{o n}$ if our theoretical analysis process is correct, which will be verified in numerical results. According to Little's Formula, the average waiting time for CU can be obtained by

$$
E[W]=\frac{E[L]}{p_{c}}
$$

Then the average sojourn time of CU, which is the duration from the instant that the CU arrives to the instant that the CU's requirement is dealt with, can be obtained by

$$
E[T]=E[W]+\frac{1}{\mu_{c}}
$$

In this paper, we define the throughput of CUs as the amount of CUs whose services are completed in unit time. If the average tolerable delay for each $\mathrm{CU}$ is denoted as $\tau_{i}, i=1,2, \cdots, E[L]$ under steady state, the throughput can be written by

$$
C=\frac{\left|\left\{\tau_{i} \mid \tau_{i} \geq E[W], i=1,2, \cdots, E[L]\right\}\right|}{E[L] E[T]}
$$

where $(|x|)$ means the total amount of elements in set $x$.

In order to improve the throughput, an access control (AC) method is proposed as following: Before access the spectrum, all CUs having transmission requirements will send their tolerable delay message to AP of cognitive system, the AP will calculate the average sojourn time according to the environment parameters and compare it with the received messages, then the order of rejected to access spectrum for the CUs whose tolerable delays excess the sojourn time will be broadcasted, the user which has received the order will no longer launch the access requirement. According to AC method, the average sojourn time can be reduced and the throughput can be improved effectively.

These accessible spectrum resources are sensed beforehand and the sensing 
results are fused at $\mathrm{AP}$ and broadcasted to CUs, then the CUs are aroused to send their requirement messages. The use of spectrum resources does not depend on the throughput in one TS. The proposed AC method has no significant differences with prior arts written in [13] [14], except the target object of AC control. The proposed AC aims at MU, while the prior one aims at DP, the evaluation results will be reflected in Figure 6 and Figure 7 of the following sections.

If a CU is served without data packets dropping, we say the CU's transmission is intact. The ratio of the amount of CUs whose transmission is intact and the amount of CUs whose transmission is not intact is defined as transmission intact ratio to evaluate the user's quality of experience. Among the CUs allowed to access spectrum, the amount of users whose transmission is intact is different for DP-based and MU-based spectrum access analysis methods. The effects based on the two analysis methods on transmission intact ratio are displayed in numerical results.

\section{Numerical Results}

We consider a CR network based on cell structure in which the channel state transition probability from " 1 " to " 0 " $\phi_{p s}=0.3$ and the CUs' arrival rate $p_{c}=0.05$. The minimum rate matrix $\boldsymbol{R}$ can be obtained in Equation (11) of Appendix A after a certain times (here is 36 ) iterations. Then we can obtain the closed-form solutions of Equation (7) to Equation (10). In order to express the throughput, the CUs with communication requirements are sorted from small to large according to tolerable delay. The smallest tolerable delay is $5 \mathrm{TS}$, then the tolerable delay is set incrementally by 1 TS.

Figure 3 verifies the validity of our queuing analysis since $\rho_{p}^{o n}=P_{p u}$ and indicates that the probability that the channel is occupied by PU will increase with $\phi_{p}$. However, the probability has no effect with the changing of service completion probability. This conforms to the principle of preemptive priority for spectrum access in CR networks. Without the consideration of interference, the activities of CUs have no impact on PUs.

Figure 4 shows the impacts of $\mu_{c}$ and $\phi_{p}$ on the average sojourn time of CUs. With the increasing of $\mu_{c}$, the average sojourn time will decrease, while with the increasing of $\phi_{p}$, the average sojourn time will increase. This is because the increasing of service completion probability can lead to a reducing of queue length, while the increasing of the probability that channel is occupied by PU will cause a reducing of the chance to access the channel for CUs. From Figure 4 we can see that if the tolerable delay before accessing the channel is given for a $\mathrm{CU}$, the related parameters can be adjusted to satisfy the requirement of the $\mathrm{CU}$, or satisfy the user losing rate under this delay requirement when parameters are fixed, according to the quantitative analysis in Figure 4 Here the user losing means the $\mathrm{CU}$ will loss the chance to access the current expected spectrum, while it can search other preferable channels or networks to access. While in the 


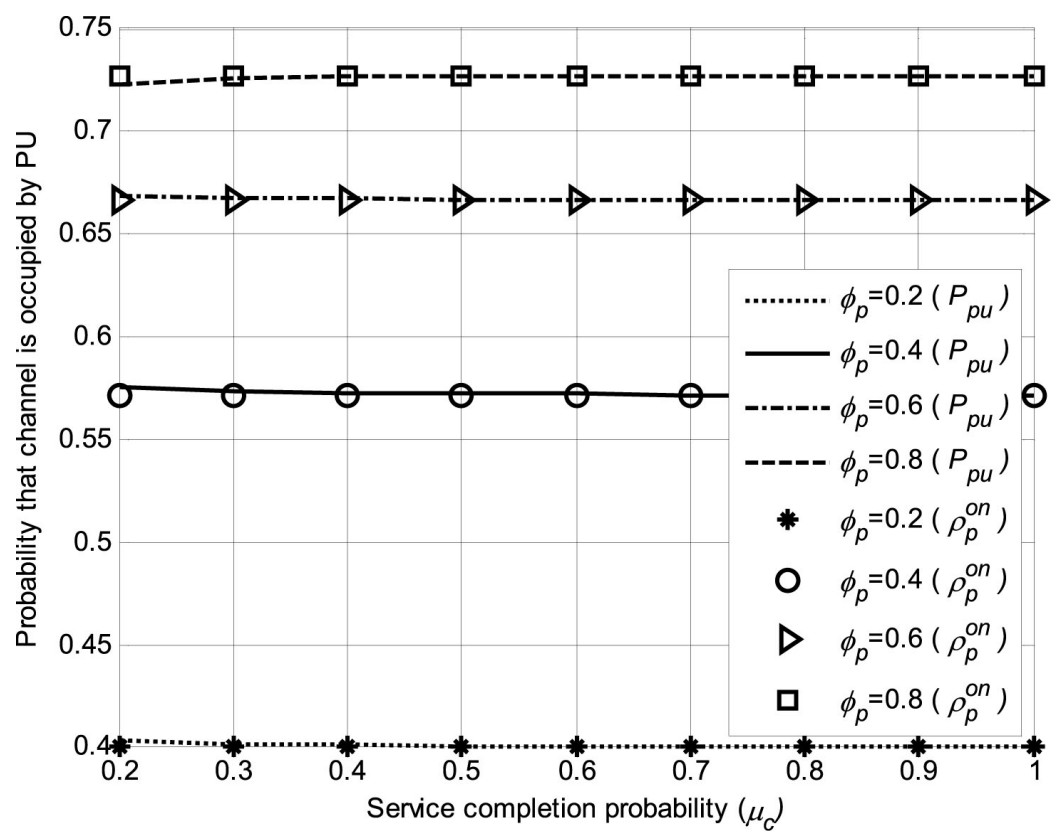

Figure 3. Effects of $\mu_{c}$ and $\phi_{p}$ on the probability that channel is occupied by PU.

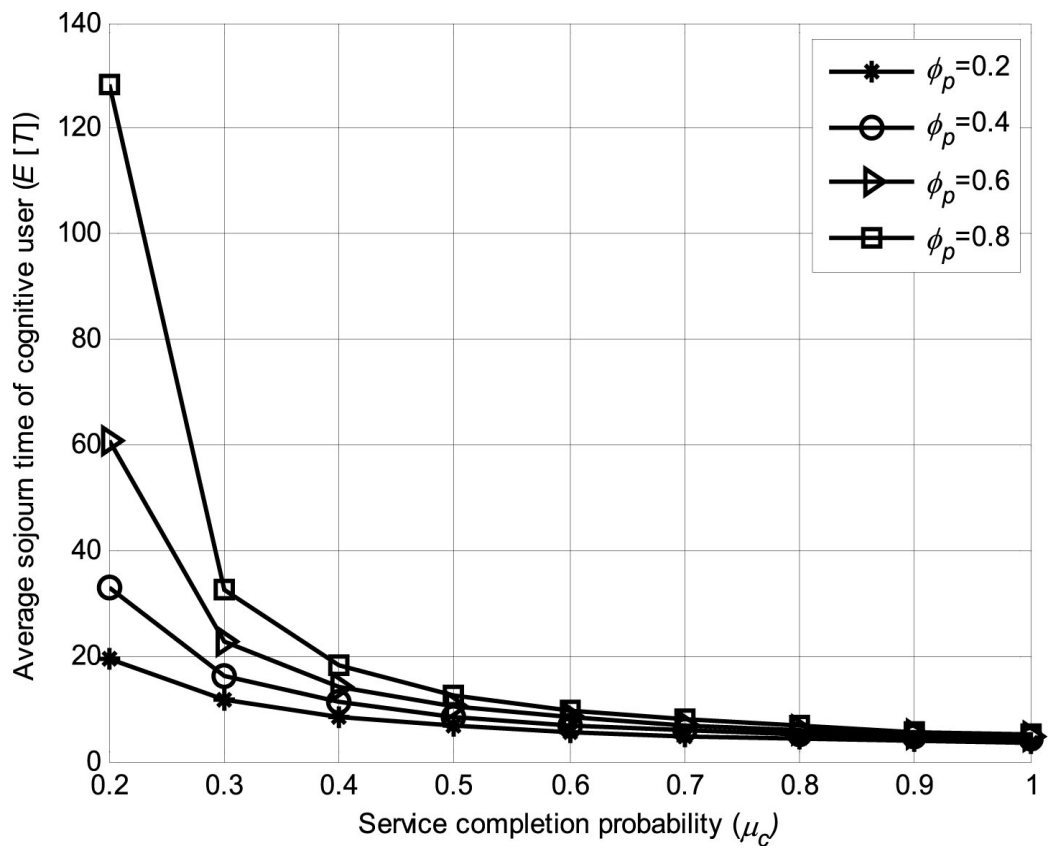

Figure 4. Impacts of $\mu_{c}$ and $\phi_{p}$ on average sojourn time.

data packet-based model, the user allowed to access the channel may be dropped during the communication which is caused by spectrum handoff, and thus this will restart the channel access process damaging the user experience seriously.

Figure 5 shows the comparison of throughput between with $\mathrm{AC}$ and without AC. First, with the increasing of $\mu_{c}$, the throughput will increase. It is easy to explain the result just as the changing trend of sojourn time in Figure 4. Furthermore, we will have a larger throughput using AC method than without using 


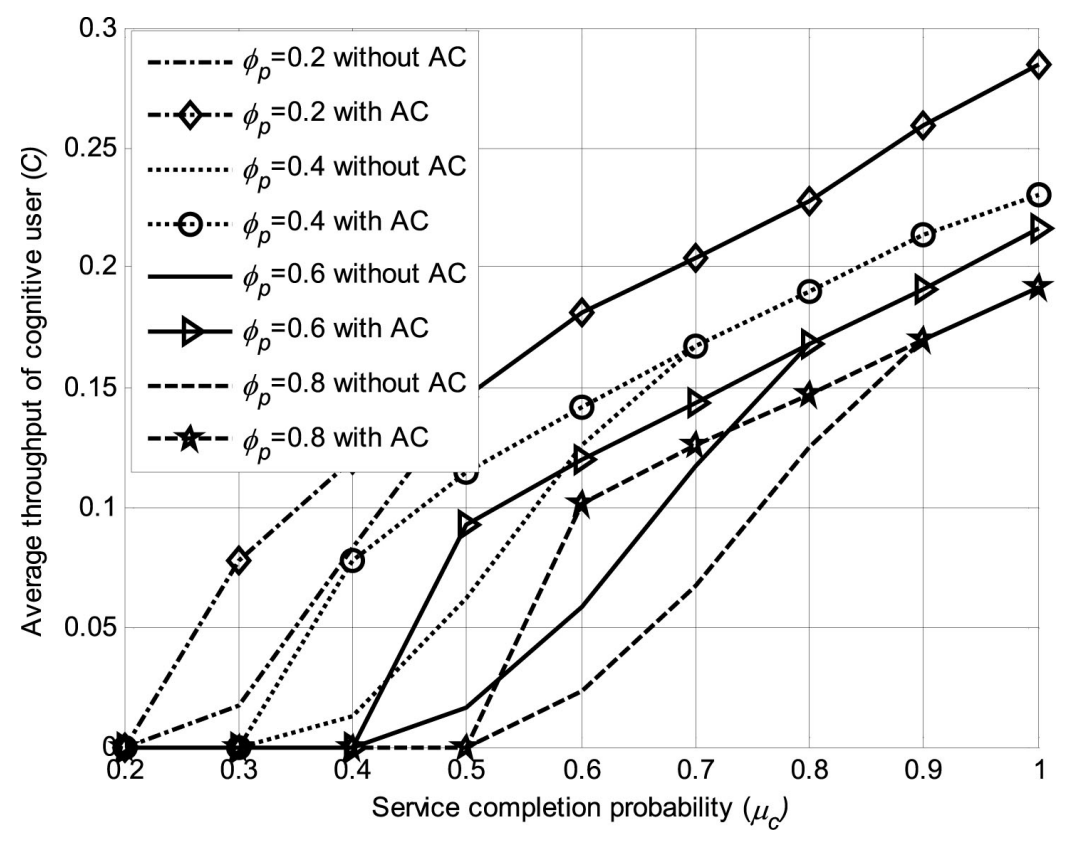

Figure 5. Differences of throughput with AC and without AC.

AC method under the same $\phi_{p}$. However, this advantage can be only reflected in a certain region of service completion probability. For example, when $\phi_{p}$ is 0.6 , the effective region of $\mu_{c}$ to improve throughput is in between 0.4 and 0.8 . Especially when the region is in between 0.5 and 0.6 , the throughput can be improved as many as three times. However, when $\mu_{c}$ is lower than 0.4 or higher than 0.8 , the AC method will have no impact on improving throughput. This is because that, when service completion probability is lower than 0.4 , maybe there is not any CUs which can tolerate the sojourn time and the throughput is zero; when service completion probability is higher than 0.8 , maybe the sojourn time can be accepted by all of the CUs and the throughput has already reached the maximum limit.

Second, with the increasing of $\phi_{p}$, the throughput decreases and the joining point of "without $\mathrm{AC}$ " and "with $\mathrm{AC}$ " postpones. Since $\phi_{p}$ is the transition probability that channel state from " 0 " to " 1 ", the value of $\phi_{p}$ increases means the probability that channel is idle decreases, which can be easily known from $\rho_{p}^{o f f}=\phi_{p s} /\left(\phi_{p s}+\phi_{p}\right)$. This will reduce the spectrum access chance. Accordingly, the sojourn time for CUs will increase and the average throughput will decrease undoubtedly. Similarly, with the increasing of $\phi_{p}$, the service completion probability has to be increased to make the throughput without AC reach that with AC.

Figure 6 and Figure 7 display the evaluation results of comparison between MU-based analysis method and DP-based analysis method. The number of data packets for each CU's is assumed as 2 . To ensure the steady state of the queuing system, we set $\mu_{c}$ from 0.2 to 0.5 which can guarantee DP's arrival probability is less than service probability. Figure 6 shows the throughput based on MU is slightly less than that based on DP. It's not too hard to understand, the CU can 


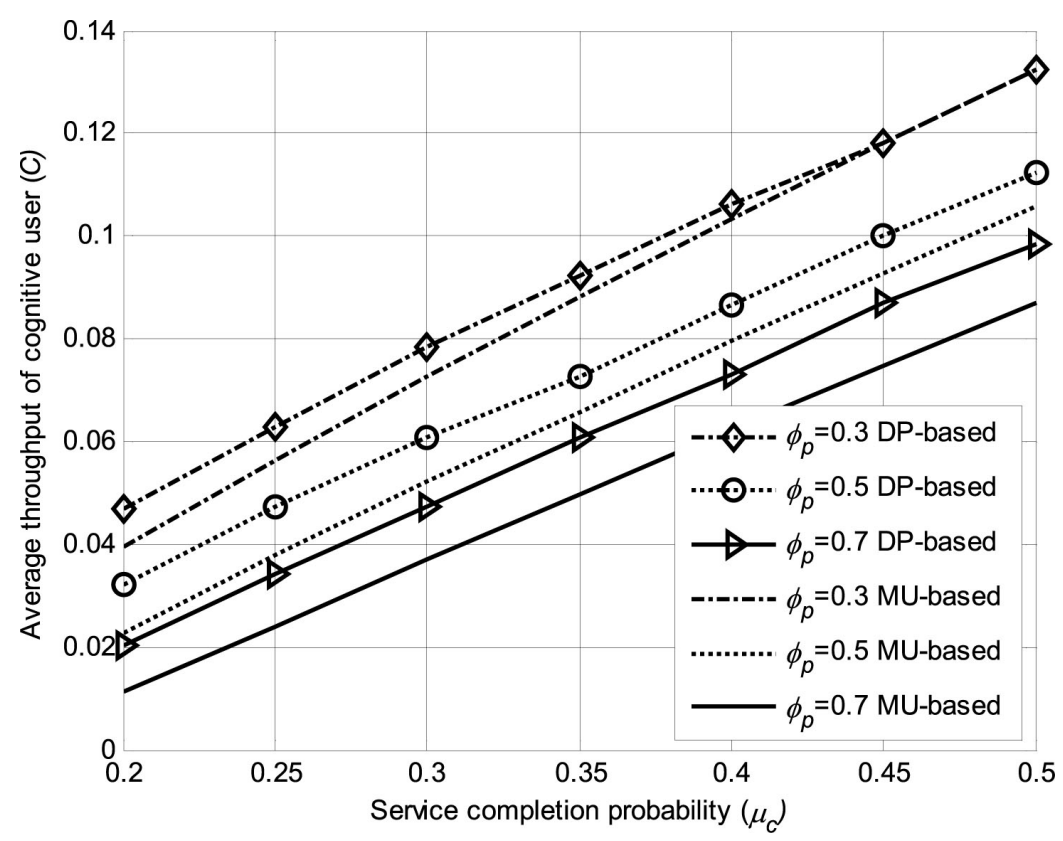

Figure 6. The throughput comparison for MU-based and DP-based analysis methods.

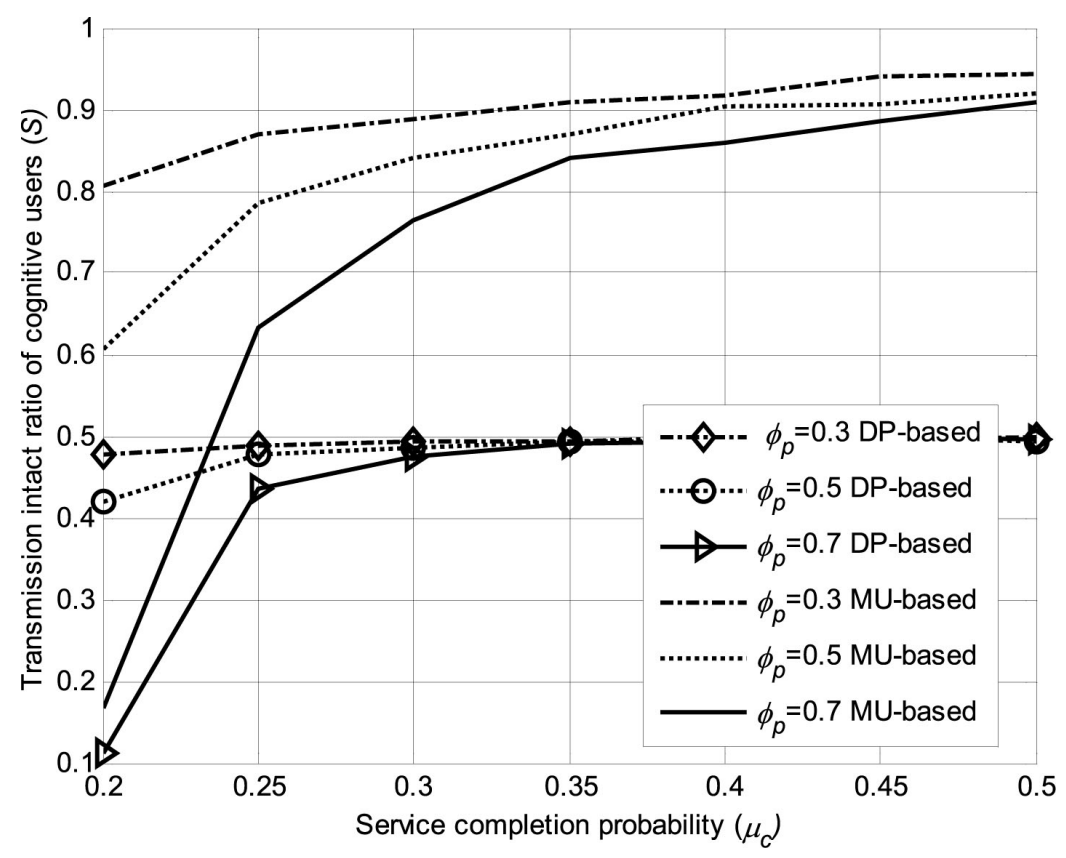

Figure 7. The transmission intact ratio comparison for MU-based and DP-based analysis methods.

have a higher probability to be allowed to access spectrum based on DP analysis. This is because only when all the data packets of a $\mathrm{CU}$ are rejected to access spectrum, will the CU be rejected. However, the transmission intact ratio of CUs based on DP is lower than that based on MU, which is shown in Figure 7. This is because if the delay limit of one of the data packets of a $\mathrm{CU}$ exceeds its sojourn time, the CU's transmission is regarded as incomplete. 


\section{Conclusion}

An opportunistic spectrum access status based on MU is analyzed in this paper. A discrete-time queuing model is established to derive the average sojourn time and throughput of cognitive users by employing the matrix-geometric solution theory. Furthermore, an access control method based on MU is proposed to improve the throughput. Numerical results confirm that the MU-based spectrum access analysis outperforms DP-based with respect to quality of experience. These results can provide the guidance at macro-level for deploying the access points and designing a spectrum access regulation in cognitive radio networks. In future work, a finite buffer capacity can be considered into the queuing model and multiple-channel network should be taken into account.

\section{Acknowledgements}

This work is supported by the National Natural Science Foundation of China (No. 61701202) and the open research fund of National Mobile Communications Research Laboratory (No. 2019D17).

\section{Conflicts of Interest}

The authors declare no conflicts of interest regarding the publication of this paper.

\section{References}

[1] Akyildiz, I.F., Lee, W., Vuran, M.C., et al. (2006) Next Generation/Dynamic Spectrum Access/Cognitive Radio Wireless Networks: A Survey. Computer Networks, 50, 2127-2159. https://doi.org/10.1016/j.comnet.2006.05.001

[2] Song, M., Xin, C., Zhao, Y. and Cheng, X. (2012) Dynamic Spectrum Access: From Cognitive Radio to Network Radio. IEEE Wireless Communications, 19, 23-29. https://doi.org/10.1109/MWC.2012.6155873

[3] Khattab, A., Perkins, D. and Bayoumi, M. (2013) Opportunistic Spectrum Access Challenges in Distributed Cognitive Radio Networks. Cognitive Radio Networks from Theory to Practice. Springer, New York.

[4] Atallah, R.F., Khabbaz, M.J. and Assi, C.M. (2015) Vehicular Networking: A Survey on Spectrum Access Technologies and Persisting Challenges. Vehicular Communications, 2, 125-149. https://doi.org/10.1016/j.vehcom.2015.03.005

[5] Kim, K.J., Kwak, K.S. and Choi, B.D. (2013) Performance Analysis of Opportunistic Spectrum Access Protocol for Multi-Channel Cognitive Radio Networks. Journal of Communications and Networks, 15, 77-86. https://doi.org/10.1109/JCN.2013.000013

[6] Hai, J., Lifeng, L., Rongfei, F., et al. (2009) Optimal Selection of Channel Sensing Order in Cognitive Radio. IEEE Transactions on Wireless Communications, 8, 297-307. https://doi.org/10.1109/T-WC.2009.071363

[7] Lee, W., Kang, J. and Kang, J. (2015) Joint Resource Allocation for Throughput Enhancement in Cognitive Radio Femtocell Networks. IEEE Wireless Communications Letters, 4, 181-184. https://doi.org/10.1109/LWC.2015.2392775

[8] Han, Y., Ekici, E., Kremo, H. and Altintas, O. (2017) Throughput-Efficient Channel 
Allocation Algorithms in Multi-Channel Cognitive Vehicular Networks. IEEE Transactions on Wireless Communications, 16, 757-770. https://doi.org/10.1109/TWC.2016.2629484

[9] Beibei, W., Zhu, J., Liu, K.J.R., et al. (2009) Primary-Prioritized Markov Approach for Dynamic Spectrum Allocation. IEEE Transactions on Wireless Communications, 8, 1854-1865. https://doi.org/10.1109/T-WC.2008.080031

[10] Won-Yeol, L. and Akyildiz, I.F. (2012) Spectrum-Aware Mobility Management in Cognitive Radio Cellular Networks. IEEE Transactions on Mobile Computing, 11, 529-542. https://doi.org/10.1109/TMC.2011.69

[11] Bhushan, S., Bali, R.S. and Kaur, A. (2015) Spectrum Hand off in Cognitive Radio Network using Dynamic Threshold. 2015 International Conference on Green Computing and Internet of Things (ICGCIoT), Noida, 8-10 October 2015, 1292-1297. https://doi.org/10.1109/ICGCIoT.2015.7380665

[12] Chang, P.-H., Chang, K.-F., Chen, Y.-C. and Ye, L.-K. (2013) Performance Analysis of Channel Switching with Various Bandwidths in Cognitive Radio. Proceedings of 12th International Conference on Networks, Seville, 27 January-1 February 2013, 29-33.

[13] Li-Chun, W., Chung-Wei, W. and Kai-Ten, F. (2011) A Queueing-Theoretical Framework for QoS-Enhanced Spectrum Management in Cognitive Radio Networks. IEEE Wireless Communications, 16, 18-26.

[14] Wang, L., Wang, C. and Chang, C. (2012) Modeling and Analysis for Spectrum Handoffs in Cognitive Radio Networks. IEEE Transactions on Mobile Computing, 11, 1499-1513. https://doi.org/10.1109/TMC.2011.155

[15] Rashid, M., Hossain, M., Hossain, E., et al. (2009) Opportunistic Spectrum Scheduling for Multiuser Cognitive Radio: A Queueing Analysis. IEEE Transactions on Wireless Communications, 8, 5259-5269. https://doi.org/10.1109/TWC.2009.081536

[16] Tian, N., Xu, X. and Ma, Z. (2008) Discrete-Time Queuing Theory. 1st Edition, Science Press, Beijing.

[17] Neuts, M.F. (1981) Matrix-Geometric Solutions in Stochastic Models: An Algorithmic Approach. The Johns Hopkins University Press, Baltimore and London. 


\section{Appendix A}

Referring to theorem 12.1 of [17], if $p_{c}<\mu_{c}$, Markov transition probability matrix $\tilde{\boldsymbol{P}}$ is positive recurrence, the process can reach steady state, then we have:

1) The smallest non-negative solution of the following equation exists, which is regarded as rate matrix $\boldsymbol{R}$

$$
\boldsymbol{X}=\boldsymbol{X}^{2} \boldsymbol{B}_{0}+\boldsymbol{X}^{1} \boldsymbol{B}_{1}+\boldsymbol{B}_{2}
$$

2) For $i \geq 2$, we have

$$
\pi_{i}=\pi_{1} \cdot \boldsymbol{R}^{i-1}
$$

3) $\boldsymbol{\pi}_{0}, \boldsymbol{\pi}_{1}$ are normal left invariant eigenvectors of $\boldsymbol{B}[\boldsymbol{R}]$, which satisfy

$$
\left(\pi_{0}, \pi_{1}\right) \boldsymbol{B}[\boldsymbol{R}]=\left(\pi_{0}, \pi_{1}\right)
$$

where $\boldsymbol{B}[\boldsymbol{R}]=\left[\begin{array}{cc}\tilde{\boldsymbol{A}}_{0} & \tilde{\boldsymbol{A}}_{1} \\ \tilde{\boldsymbol{B}}_{0} & \boldsymbol{R} \tilde{\boldsymbol{B}}_{0}+\tilde{\boldsymbol{B}}_{1}\end{array}\right]$, and the normalized equation written as the second formula of Equation (6) can be rewritten by

$$
\left[\pi_{0}+\pi_{1}(\boldsymbol{I}-\boldsymbol{R})^{-1}\right] \boldsymbol{e}=1
$$

Then, $\pi_{0}, \pi_{1}$ can be obtained by solving Equations (13) and (14) simultaneously.

The matrix sequences $\{\boldsymbol{X}(N), N \geq 0\}$ will be given by substituting the initialization $\boldsymbol{X}(0)=\mathbf{0}$ into Equation (11) and repeated iteration. The dominated convergence theorem has verified that these matrix sequences will converge to a non-negative matrix which is just $\boldsymbol{R}$ regarded as rate matrix in queuing theory. Finally, by substituting rate matrix $\boldsymbol{R}$ into Equation (12), the steady state probability $\pi$ will be obtained. 